\title{
CALCULATION OF CORRELATION EFFECTS FOR GRAIN-BOUNDARY AND DISLOCATION-PIPE SELF-DIFFUSION
}

\section{by}

J. T. Robinson and N. L. Peterson

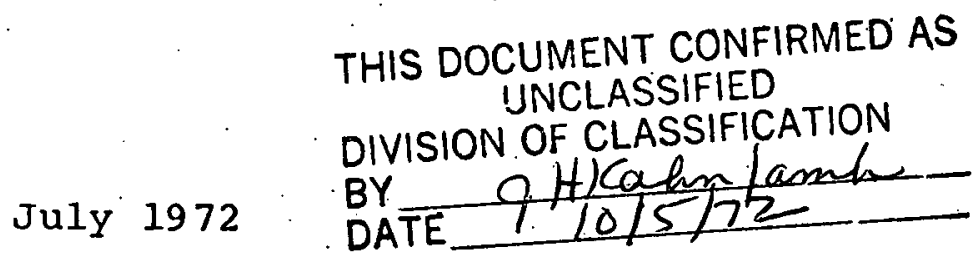

Cornell University

Ithaca, New York 14850

Report \#1838

Issued by

The Materials Science Center

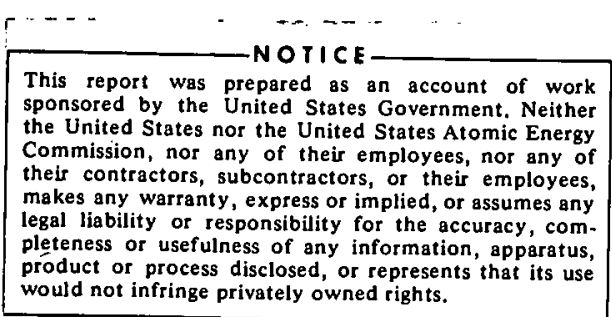




\section{DISCLAIMER}

This report was prepared as an account of work sponsored by an agency of the United States Government. Neither the United States Government nor any agency Thereof, nor any of their employees, makes any warranty, express or implied, or assumes any legal liability or responsibility for the accuracy, completeness, or usefulness of any information, apparatus, product, or process disclosed, or represents that its use would not infringe privately owned rights. Reference herein to any specific commercial product, process, or service by trade name, trademark, manufacturer, or otherwise does not necessarily constitute or imply its endorsement, recommendation, or favoring by the United States Government or any agency thereof. The views and opinions of authors expressed herein do not necessarily state or reflect those of the United States Government or any agency thereof. 


\section{DISCLAIMER}

Portions of this document may be illegible in electronic image products. Images are produced from the best available original document. 
CALCULATION OF CORRELATION EFFECTS FOR GRAIN-BOUNDARY.

AND DISLOCATION-PIPE SELF-DIFFUSION *

\author{
by . \\ J. T. Robinson ${ }^{* *}$ and N. L. Peterson ${ }^{* * *}$ \\ ** Cornell University \\ Materials Science and Engineering \\ Ithaca, New York 14850 \\ $\star \star \star *$ \\ Materials Science Division \\ Argonne National Laboratory \\ Argonne, Illinois 60439
}

\begin{abstract}
The correlation factor for grain-boundary and dislocation-pipe self-diffusion was calculated for a vacancy mechanism by assuming the applicability of the geometric model previously formulated by the authors. The correlation factor is temperature dependent and substantially lower than that for lattice selfdiffusion due to correlated vacancy motion. The calculations and experimental isotope effects for silver are consistent with a vacancy-dislocation (or grain boundary) binding energy that decreases 0.05-0.10 eV per atomic spacing away from the dislocation or grain boundary and many-body saddle-point interactions which are greater than those for lattice self-diffusion via vacancies.
\end{abstract}

* This work was supported by the U. S. Atomic Energy Commisson. 


\section{INTRODUCTION}

The problem of resolving the mechanism for grain-boundary self-diffusion has received considerable attention during the past few years. The experiments of Cannon and Stark (1)' and Volin, Lie, and Balluffi(2) and subsequent analysis by Balluffi(3) have shown that measurements of grain-boundary self-diffusion coefficients in face-centered-cubic ( $f c c$ ) metals are consistent with a vacancy mechanism. However, as in the case of lattice diffusion, measurements of only the self-diffusion coefficient do not conclusively determine the diffusion mechanism. Analysis of measurements of the isotope effect, $f \Delta K$, with respect to theoretical calculations of correlation factors are desirable for a more complete resolution of the problem. The isotope-effect parameters are defined as $f$ the correlation factor, and $\Delta K$ the fraction of kinetic energy possessed by the diffusing atom in the saddle-point configuration in the jump direction.

Robinson and Peterson (4) have measured the isotope effect for grain-boundary self-diffusion in silver. Comparison of the f' $\Delta K^{\prime}$ measurements with first-order calculations of the correlation factor was consistent with a vacancy mechamism for grain-boundary self-diffusion. [Note: The prime mark implies the parameters appropriate for grain-boundary diffusion.]

The major question regarding this analysis is the validity of the theoretical values of the correlation factor. The method of Leclaire and Lidiard ${ }^{(5)}$ was employed with questionable validity, because the atomic geometry assumed near a grain boundary or dislocation does not yield atomic jumps along directions of two- 
fold rotational symmetry. Numerical results using the technique described gave correlation factors that.were dependent upon the initial tracer jump. However, it was concluded that the isotopeeffect measurements and correlation-factor calculations were consistent with a vacancy mechanism for grain-boundary self-diffusion in fcc metals.

The purpose of the present paper is to report a more realistic calculation of the correlation factor for grain-boundary self-diffusion along dislocation cores that may be found in lowangle. $\langle 100\rangle$ symmetric tilt boundaries. The geometric model is that proposed by Robinson and Peterson; (4) the calculations are performed using Howard's ${ }^{(6)}$ general matrix method. The correlation factors that result from these calculations for a vacancy mechanism are compared with the experimental isotope effects for grainboundary self-diffusion in silver polycrystals and $\langle 100\rangle$ tilt boundaries. Since the results and subsequent interpretation are model dependent, the major properties of the geometric model are given.

\section{MODEL}

The atomic structure assumed for the compressed region of a dislocation in a $\langle 100\rangle$ low-angle symmetric tilt boundary is shown in Fig. 1. Details of the motivation for using such a structure are given in Ref. 4. The crosshatched circles represent sites along the last row of atoms in the extra half plane of the edge dislocation. These are designated as type-1 sites and are expected to be positions of maximum vacancy-dislocation binding energy. (7) The other sites shown in.Fig. 1 are type-2 sites and are nearest neigh- 
bors to the positions where the maximum binding energy occurs. The only other sites considered in the calculations are designated as type-3, which are nearest neighbors to type- 2 sites. The vacancy-dislocation binding energy decreases with distance from type1 sites.

The vacancy-dislocation binding energy and the structure of the model imply that the atom-vacancy exchange frequencies are position dependent. Throughout the calculations it is assumed that atom-vacancy exchanges are possible only between nearest-neighbor positions. Therefore all tracer jumps with nonzero $x$-displacement have identical $x$-projections. A jump dependent $x$-projection would change the value of the correlation factor; however, uncertainties in the atomic structure near a grain-boundary or dislocation disallow reasonable estimates of the position dependence of the tracer jump vectors. Tables 1 and 2 give the definitions of the vacancy jump frequencies and possible jump frequencies from the individual sites.

\section{METHOD OF CALCULATION}

Howard's method of calculating correlation factors is applicable to atomic jumps that are along directions of low crystal symmetry. Since the number of sites in the unit cell shown in Fig. 1 is small, all sites are considered unique. Furthermore, all. tracer jumps with nonzero $\mathrm{x}$-displacements are assumed to be unique. It should be noted that these assumptions of uniqueness are not absolutely necessary since some sites and jumps are equivalent. The equivalence relationships will only reduce the size of the matrices employed. 
The fundamental equation of the method is

$$
\mathrm{f}_{\mathrm{x}}=1+2 \hat{\mathrm{C}} \cdot \hat{\mathrm{T}}_{1}\left(\hat{I}-\hat{\mathrm{T}}_{1}\right)^{-1} \cdot \hat{\mathrm{I}}
$$

where $f_{x}$ is the correlation factor due to atomic jumps with nonzero displacements in the $x$-direction. The term $\hat{c}$ is a row matrix whose $\mathrm{N}$ components, $c_{\alpha}$, give the fraction of $\alpha$-type jumps in any long sequence of jumps, and $\mathrm{N}$ is the number of unique tracer jumps. The term $\hat{\mathrm{T}}_{1}$ is a $\mathrm{N}$ by $\mathrm{N}$ matrix with components

$$
t_{1}^{\alpha \beta}=p_{1+}^{\alpha \beta}-p_{1-}^{\alpha \beta}
$$

where $p_{1 \pm}^{\alpha \beta}$ are equal to the probability that, given an initial $\alpha-$ type jump, the next tracer jump is a $\beta$ type and has an $x$-projection in the same $(+)$. or opposite (-) direction as that of the initial jump. The term $\hat{I}$ is the $N$ by $N$ unit matrix, and $\hat{I}$ is the unit column matrix. The components $t_{l}^{\alpha \beta}$ are obtained by direct calculation of the $p_{1 \pm}^{\alpha \beta}$

$$
\begin{aligned}
& \text { The terms } p_{1 \pm}^{\alpha \beta} \text { are given by } \\
& p_{l \pm}^{\alpha \beta}=p_{j \pm}^{\alpha} b_{j \pm}^{\beta},
\end{aligned}
$$

where ${ }_{j \neq}^{\alpha}$ is the probability that, given an initial $\alpha$-type jump, the vacancy exists at a type-j site, which causes a $\beta$-type tracer jump with $x$-projection in the same $(+)$ or opposite (-) direction as the $\alpha$ jump. The term $b_{j \pm}^{\beta}$ is the probability that the tracer and the vacancy at site $j$ exchange to give a $\beta$-type jump with the same (+) or opposite $(-) x$-projection as the initial tracer jump. The $b_{j \pm}^{\beta}$ are obtained by inspection. The terms $\mathrm{P}_{j \pm}^{\alpha}$ are $j^{\text {th }}$ components of $\mathrm{N}$ column matrices, $\hat{\mathrm{P}}^{(\alpha)}$. The $\hat{\mathrm{P}}^{(\alpha)}$ are calculated from

$$
\hat{\mathrm{P}}^{(\alpha)}=(\hat{I}-\hat{\mathrm{A}})-1 \cdot \hat{\mathrm{P}}_{0}^{(\alpha)},
$$

where $\hat{A}$ is an $M$ dimensional square matrix with components $A_{i j}$. that 
give the probability that the next vacancy jump from site $j$ is to a type $i$ site. The term $\hat{\mathrm{P}}_{0}^{(\alpha)}$ is a $M$ component column vector with components that give the vacancy distribution immediately after an a-type jump, which leaves the tracer at the origin.

By using Tables 1 and 2 in conjunction with Fig. 1 , it is possible to determine the components of $\hat{A}$ and $\hat{c}$ in terms of five jump frequency ratios:

$$
\begin{aligned}
& \delta_{1}=\mathrm{w}_{3} / \mathrm{w}_{2} \\
& \delta_{2}=\mathrm{k} / \mathrm{w}_{2} \\
& \delta_{3}=\mathrm{w}_{2}^{\prime} / \mathrm{w}_{1} \\
& \delta_{4}=\mathrm{k}^{\prime} / \mathrm{w}_{1} . \\
& \delta_{5}=\mathrm{w}_{1} / \mathrm{w}_{2} .
\end{aligned}
$$

The only jumps that have nonzero $x$-displacements are of the type which place the tracer at $(0,0,0)$ and the vacancy at one of the sites given by $(1,0,1),(1,0,-1),(1,-1,0),(1,1,0),(-1,0,1)$, $(-1,0,-1),(-1,-1,0)$ and $(-1,1,0)$. The term $\hat{\mathrm{c}}$ is given by

$$
\begin{aligned}
c_{\Sigma} \hat{c}= & {\left[1 /\left(6+2 \delta_{5}\right), 1 /\left(2+6 / \delta_{5}\right), 1 /\left(6+2 \delta_{5}\right), 1 /\left(6+2 \delta_{5}\right), 1 /\left(6+2 \delta_{5}\right),\right.} \\
& \left.1 /\left(2+6 / \delta_{5}\right), 1 /\left(6+2 \delta_{5}\right), 1 /\left(6+2 \delta_{5}\right)\right],
\end{aligned}
$$

where the factor $c_{\Sigma}$ accounts for the requirement that

$$
\sum_{\alpha=1}^{N} c_{\alpha}=1 \text {. }
$$

The matrix $\hat{A}$ is given by 


$\hat{A}=\left(\begin{array}{cccccccccccc}0 & 0 & 0 & 0 & 1 / X & 1 / X & 0 & 0 & 0 & 0 & 1 / Y & 1 / Y \\ 0 & 0 & 0 & 0 & 0 & 0 & 1 / X & 1 / X & 0 & 0 & 1 / Y & 1 / Y \\ 0 & 0 & 0 & 0 & 0 & 0 & 1 / X & 1 / X & 1 / W & 1 / W & 0 & 0 \\ 0 & 0 & 0 & 0 & 1 / X & 1 / X & 0 & 0 & 1 / W & 1 / W & 0 & 0 \\ 1 / Z & 0 & 0 & 1 / X & 0 & 0 & 0 & 0 & 0 & 1 / W & 1 / Y & 0 \\ 1 / Z & 0 & 0 & 1 / X & 0 & 0 & 0 & 0 & 1 / W & 0 & 0 & 1 / Y \\ 0 & 1 / Z & 1 / X & 0 & 0 & 0 & 0 & 0 & 1 / W & 0 & 0 & 1 / Y \\ 0 & 1 / Z & 1 / X & 0 & 0 & 0 & 0 & 0 & 0 & 1 / W & 1 / Y & 0 \\ 0 & 0 & 1 / Q & 1 / Q & 0 & 1 / Q & 1 / Q & 0 & 0 & 0 & 0 & 0 \\ 0 & 0 & 1 / Q & 1 / Q & 1 / Q & 0 & 0 & 1 / Q & 0 & 0 & 0 & 0 \\ 1 / Z & 1 / Z & 0 & 0 & 1 / X & 0 & 0 & 1 / X & 0 & 0 & 0 & 0 \\ 1 / Z & 1 / Z & 0 & 0 & 0 & 1 / X & 1 / X & 0 & 0 & 0 & 0 & 0\end{array}\right)$,

where $\mathrm{x}=5+3 / \delta_{1}+4 \delta_{2} / \delta_{1}$

$$
\begin{aligned}
& \mathrm{Y}=6+2 / \delta_{1}+4 \delta_{2} / \delta_{1} \\
& \mathrm{Z}=4+1 / \delta_{1}+7 \delta_{2} / \delta_{1} \\
& \mathrm{~W}=6+2 / \delta_{3}+4 \delta_{4} / \delta_{3} \\
& \mathrm{Q}=3+5 \delta_{1}+4 \delta_{2}
\end{aligned}
$$

The initial vacancy distribution after an $\alpha$-type jump corresponds to a vacancy at those equivalent sites possibly occupied by a vacancy after an $\alpha$-type jump has occurred. All components of $P_{0}(\alpha)$, $\alpha=1,2, \ldots 8$, are zero except the component corresponding to the site occupied by the vacancy after the $\alpha$ jump places the tracer at the origin. In all cases, the value of the nonzero component is unity. Because all sites and jumps are considered unique, the $b_{j \pm}^{\beta}$ are probabilities that the vacancy jumps to the origin from sites giving a $\beta$-type jump. These nonzero terms are 


$$
\begin{aligned}
& b_{11 \pm}^{1}=1 /\left(2+6 \delta_{1}+4 \delta_{2}\right) \\
& b_{10 \pm}^{2}=1 /\left(2+6 \delta_{3}+4 \delta_{4}\right) \\
& b_{8 \pm}^{3}=1 /\left(3+5 \delta_{1}+4 \delta_{2}\right) \\
& b_{5 \pm}^{4}=1 /\left(3+5 \delta_{1}+4 \delta_{2}\right) \\
& b_{12 \pm}^{5}=1 /\left(2+6 \delta_{1}+4 \delta_{2}\right) \\
& b_{9 \pm}^{6}=1 /\left(2+6 \delta_{3}+4 \delta_{4}\right) \\
& b_{7 \pm}^{7}=1 /\left(3+5 \delta_{1}+4 \delta_{2}\right) \\
& b_{6 \pm}^{8}=1 /\left(3+5 \delta_{1}+4 \delta_{2}\right) .
\end{aligned}
$$

The. site-to-site vacancy-dislocation binding energy differences allow numerical evaluation of the jump frequency ratios given by Eq. (5). Assuming a constant migration energy and entropy factor,

$$
\frac{w_{i}}{w_{j}}=e^{-\left(\Delta E_{i}-\Delta E_{j}\right) / k_{B} T} ;
$$

where $w_{i}$ is the jump frequency, $\Delta E_{i}$ is the change in vacancy energy due to the jump specified by $w_{i}$, and $k_{B}$ and $T$ have their usual meanings. The term "binding energy" used throughout this paper is defined in the same sense as given in Ref. 4.

\section{RESULTS}

The results of the calculations of $f_{x}^{\prime}$ are given in Table 3. The binding conditions are chosen to represent a spectrum of values one might expect as the degree of correlation of vacancy motion changes.

The value of $\mathrm{f}_{\mathrm{x}}^{\prime}$ for the case of no vacancy-dislocation in- 
teractions is in good agreement with the value found by Leclaire and Lidiard for self-diffusion in fcc lattices. (5) The value of 0.818 compared with the true value of $0.782^{(8)}$ indicates that the model is restrictive in the sense that the number of near-neighbor shells included is somewhat small. However, the finite size of the model introduces an inherent error of only 5\%. Because of correlated vacancy motion near the dislocation, the number of sites considered would not be expected to be as important as in the case of lattice self-diffusion. Also, the results indicate that the temperature dependence and magnitude of the correlation factor are dependent upon the magnitude and rate of decrease of binding energy with distance from the dislocation core.

The results given in Table 3 are not expected to apply to all types of grain boundaries in fcc metals since the boundary structure is sensitive to the orientations of the boundary plane and single crystalline regions on either side of the boundary.

\section{DISCUSSION}

For a meaningful interpretation of the model and the results given in Table 3 , it is first necessary to relate the properties of the lattice self-diffusion isotope effect to those properties one could reasonably expect for grain-boundary and dislocationpipe self-diffusion.

Isotope-effect measurements for lattice self-diffusion in fcc material have established that diffusion occurs by means of a monovacancy mechanism, with possible divacancy contributions near the melting point. $(9-11)$ Also, $\Delta \mathrm{K}$ is independent of temperature and $0.85<\Delta \mathrm{K}<1$. Calculations of $\Delta \mathrm{K}$ by Achar ${ }^{(12)}$ agree with a temp- 
erature-independent $\Delta \mathrm{K}$ and are in reasonable agreement with the experimental results; $\Delta \mathrm{K}$ (calculated) for silver is 0.80 and $\Delta \mathrm{K}$ (experimental) is 0.92 if divacancy contributions are negligible at Low temperature ${ }^{(11)}$ and 0.96 if divacancy contributions are included. (13)

We will assume that $\Delta K^{\prime}$ for grain-boundary diffusion is similar to, but not identical with, $\Delta \mathrm{K}$ for lattice diffusion. There is reason to believe that $\Delta \mathrm{K}^{\prime}$ is somewhat smaller than $\Delta \mathrm{K}$, and, if $\Delta \mathrm{K}^{\prime}$ is temperature dependent, it decreases as the temperature increases. Near a grain boundary, lattice nearest-neighbor relations are not preserved, and the structure of the grain boundary is not close packed as in the lattice. The motions of saddle-point atoms in the lattice-diffusion process are constrained by nearest-neighbor interactions; therefore, $\Delta \mathrm{K}$ is near unity and temperature independent. It is possible that the vibrational amplitude of the atoms increases more with temperature in the open structure of grain boundaries than in the lattice. This would result in larger saddlepoint atom interactions with increasing temperature. Thus, a decrease in $\Delta \mathrm{K}^{\prime}$ as the temperature increases is not inconceivable. Also, an increase in saddle-point interactions in the diffusion direction would result in $\Delta \mathrm{K}^{\prime}<\Delta \mathrm{K}$ regardless of the temperature dependence. For the purpose of comparing the calculations of $f_{x}^{\prime}$ with experimental values of $f^{\prime} \Delta K^{\prime}$, we will assume $\Delta K^{\prime}$ is $\sim 0.75$. In Ref. 4, $\Delta K^{\prime}$ was assumed $\sim 0.85$; there is considerable uncertainty in this value of $\Delta K^{\prime}$ since the calculations of $f^{\prime}$ were questionable. The more realistic calculations given in this paper justify a lower $\Delta \mathrm{K}^{\prime}$ if calculations of $\mathrm{f}^{\prime}$ and estimates of $\Delta \mathrm{K}^{\prime}$ reasonably approximate 
the experimental isotope effects.

Figure 2 shows the relationship between experimental isotope effects for silver grain-boundary self-diffusion ${ }^{(4)}$ and predicted isotope effects using the correlation factors from Table 3 and $\Delta K^{\prime}=0.75$. The dashed line represents the average experimental value of 0.46 for silver. [Note: Previously it was determined that to within experimental uncertainty the isotope effects were identical for grain-boundary self-diffusion in silver polycrystals and $16^{\circ}<100>$ symmetric tilt bicrystals (see Ref. 4). The same reasoning is implied throughout this paper.] Regardless of the estimated value of $\Delta \mathrm{K}^{\prime}$, the binding condition $0.4,0.0$, and $0.0 \mathrm{eV}$ is unacceptable. From Table 3 and Fig. 2, this condition requires the nonphysical condition $\Delta K^{\prime}>1$. This confirms the deduction given in Ref. 4 that dislocation-pipe diffusion does not occur predominately by atomic motion along a single row of atoms in the dislocation core. The binding conditions $0.4,0.2$, and $0.1 \mathrm{eV}$ and $0.20,0.05$, and $0.05 \mathrm{eV}$ give unacceptable isotope-effect predictions. A $\Delta \mathrm{K}^{\prime}>1$ is required at the lower temperature to match the calculated values to the experimental average.

The binding conditions $0.20,0.10$, and $0.05 \mathrm{eV}$ and $0.20,0.15$, and $0.10 \mathrm{eV}$ both give physically acceptable values for $\Delta \mathrm{K}^{\prime}$. A temperature-dependent $\Delta \mathrm{K}^{\prime}$, approximately linear in $(1 / T)$, gives $\Delta \mathrm{K}^{\prime}=$ 0.70 at $623^{\circ} \mathrm{K}$, and $\Delta \mathrm{K}^{\prime}=0.76$ at $823^{\circ} \mathrm{K}$ if the correlation factor from the binding condition $0.20,0.15$, and $0.10 \mathrm{eV}$ is used to fit the average experimental isotope effect. A similar analysis for the binding condition $0.20,0.10$, and $0.05 \mathrm{eV}$ gives $\Delta \mathrm{K}^{\prime}=0.97$ at $623^{\circ} \mathrm{K}$, and $\Delta K^{\prime}=0.85$ at $823^{\circ} \mathrm{K}$. Thus, it is apparent that a unique binding 
condition and temperature-dependent f' cannot be determined without further knowledge of the magnitude of $\Delta \mathrm{K}^{\prime}$.

However, the nonuniqueness of calculated $f^{\prime}$ does not substantially distract from the essential features of grain-boundary and dislocation-pipe diffusion. The binding conditions $0.20,0.15$, and $0.10 \mathrm{eV}$ and $0.20,0.10$, and $0.05 \mathrm{eV}$ are not substantially different. Both give the correlation factors significantly lower than those for lattice self-diffusion in fcc lattices. Within the framework of the geometric model, the exact calculations of f' prove that the vacancy-dislocation binding energy decreases $0.05-0.10 \mathrm{eV}$ per nearest-neighbor spacing away from the dislocation or grain boundary in the grain boundaries in silver where the isotope-effect measurements apply. It should be noted that the values of $f^{\prime}$ which result from the binding condition $0.20,0.15$, and $0.10 \mathrm{ev}$ are preferred because a more reasonable $\Delta \mathrm{K}^{\prime}$ results.

Inasmuch as the geometric model restricts tracer and vacancy motion to a small region near the dislocation or grain boundary, the calculation of $f^{\prime}$ gives the effective grain-boundary width or dislocation core diameter for diffusion. The agreement shown in Fig. 2 for calculated $f^{\prime}$, predicted $\Delta \mathrm{K}^{\prime}$, and experimental isotope effects of grain boundaries in silver implies that fast self-diffusion occurs within two to four lattice parameters around a grain boundary or dislocation. 


\section{REFERENCES}

1. R. F. Cannon and J. P. Stark, J. Appl. Phys. 40, 4361 (1969).

2. T. E. Volin, K. H. Lie, and R. W. Balluffi, Acta Met. 19, 263 (1971).

3. R. W. Balluffi, Phys. Status Solidi 42, 11 (1970).

4. J. T. Robinson and N. L. Peterson, Surface Science 31,586 (1972) .

5. A. D. LeClaire and A. B. Lidiard, Phil. Mag. 1, 518 (1956).

6. R. E. Howard, Phys. Rev. 144, 650 (1966).

7. M. Doyama and R.M.J. Cotterill, Suppl. Japan Inst. Metals 9 , 55 (1968).

8. K. Compaan and Y. Haven, Trans. Faraday Soc. ⒉ 786 (1956).

9. N. L. Peterson, Phys. Rev. 136, A568 (1964).

10. S.J. Rothman and N. L. Peterson, Phys. Status Solidi 35, 305 (1969).

11. S. J. Rothman, N. L. Peterson, and J. T. Robinson, Phys. Status Solidi 39,635 (1970).

12. B. N. Narahari Achar, Phys. Rev. B2, 3848 (1970).

13. H. Mehrer and A. Seeger, Phys. Status Solidi 39, 646 (1970): 
Table 1. Definitions of vacancy jump frequencies

\begin{tabular}{ccc}
$\begin{array}{c}\text { Initial site } \\
\text { type }\end{array}$ & $\begin{array}{c}\text { Final site } \\
\text { type }\end{array}$ & $\begin{array}{c}\text { Jump frequency } \\
\text { designation }\end{array}$ \\
\hline 1 & 1 & $\mathrm{w}_{1}$ \\
1 & 2 & $\mathrm{k}^{\prime}$ \\
1 & 1 & $\mathrm{w}_{2}^{\prime}$ \\
2 & $3^{\mathrm{a}}$ & $\mathrm{w}^{\prime}$ \\
2 & 3 & $\mathrm{w}_{3}$
\end{tabular}

a The site 3 here refers to positions in the dilatation region of the dislocation that can be reached from a type-l site. The binding of a vacancy at these sites is assumed to be identical to that for type-3 sites in the compression region. 
Table 2. Vacancy jump frequencies

Vacancy position

$$
(0,1,1)
$$

$(0,-1,1)$

$(0 ;-1,-1)$

$(0,1,-1)$

$(1,1,0)$

$(-1,1,0)$

$(-1,-1,0)$

$(1,-1,0)$

$(-1,0,-1)$

$(1,0,-1)$

$(1,0,1)$

$(-1,0,1)$
Jump frequencies

$\mathrm{w}_{2}, 4 \mathrm{w}_{3}, 7 \mathrm{k}$

$\mathrm{w}_{2}, 4 \mathrm{w}_{3}, 7 \mathrm{k}$

$3 \mathrm{w}_{2}, 5 \mathrm{w}_{3}, 4 \mathrm{k}$

$3 w_{2}, 5 w_{3}, 4 k$

$3 \mathrm{w}_{2}, 5 \mathrm{w}_{3}, 4 \mathrm{k}$

$3 \mathrm{w}_{2}, 5 \mathrm{w}_{3}, 4 \mathrm{k}$

$3 \mathrm{w}_{2}, 5 \mathrm{w}_{3}, 4 \mathrm{k}$

$3 \mathrm{w}_{2}, 5 \mathrm{w}_{3}, 4 \mathrm{k}$

$2 w_{1}, 6 w_{2}^{\prime}, 4 k^{\prime}$

$2 w_{1}, 6 w_{2}^{\prime}, 4 k^{\prime}$

$2 \mathrm{w}_{2}, 6 \mathrm{w}_{3}, 4 \mathrm{k}$

$2 w_{2}, 6 w_{3}, 4 k$ 
Table 3. Temperature and binding energy dependence of calculated correlation factors

\begin{tabular}{|c|ccccc|}
\hline $\begin{array}{c}\text { Binding } \\
\text { conditions } \\
(\mathrm{eV})\end{array}$ & \multicolumn{4}{|c}{$\mathrm{f}^{\prime}$ at the indicated temperature, ${ }^{\circ} \mathrm{K}$} \\
\cline { 2 - 6 } & 623 & 673 & 723 & 773 & 823 \\
\hline $0.00,0.00,0.00$ & 0.818 & 0.818 & 0.818 & 0.818 & 0.818 \\
$0.20,0.15,0.10$ & 0.609 & 0.624 & 0.638 & 0.649 & 0.660 \\
$0.20,0.10,0.05$ & 0.472 & 0.491 & 0.510 & 0.527 & 0.543 \\
$0.20,0.05,0.05$ & 0.420 & 0.438 & 0.455 & 0.472 & 0.488 \\
$0.40,0.20,0.10$ & 0.435 & 0.445 & 0.464 & 0.478 & 0.491 \\
$0.40,0.00,0.00$ & 0.334 & 0.335 & 0.336 & 0.337 & 0.339 \\
\hline
\end{tabular}




\section{FIGURE CAPTIONS}

Figure 1. Structural model for correlation-factor calculations:

Figure 2. Temperature dependence of the isotope effect, calculated using values from Table 3 and $\Delta \mathrm{K}^{\prime}=0.75$, - and $\circ$ are experimental data from Ref. 4 for polycrystals and $\langle 100\rangle$ tilt bicrystals of silver, ---average of the experimental data from Ref. 4. 


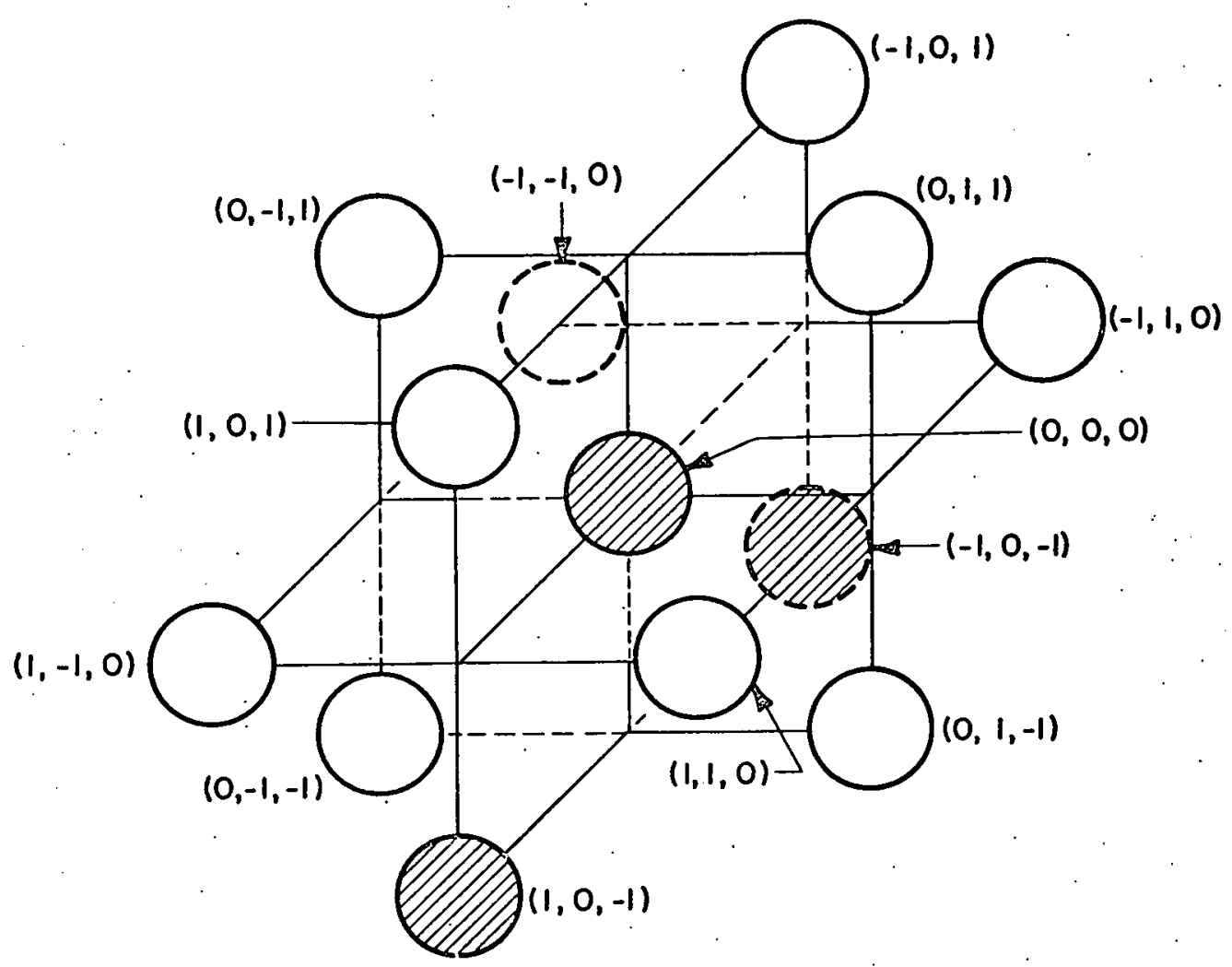

Figure 1 


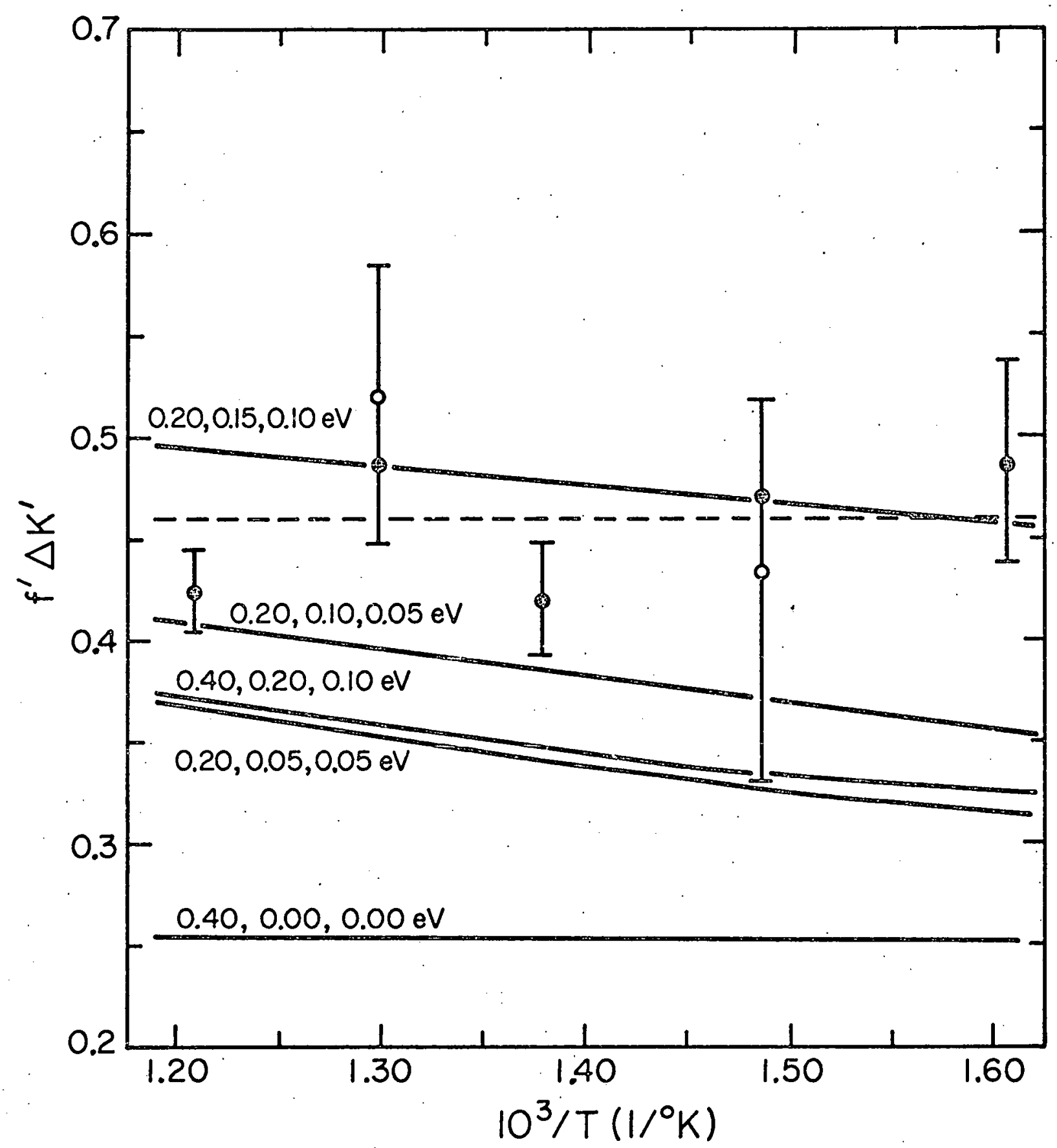

Figure 2 


\section{CALCULATION OF CORRELATION EFFECTS FOR GRAIN-BOUNDARY AND DISLOCATION-PIPE SELF-DIFFUSION}

by

J. T. Robinson and N. L. Peterson

July 1972

Cornell University

Ithaca, New York 14850

Report \#1838

Issued by

The Materials Science Center

NOIICE

This report was prepared as an account of work sponsored by the United States Government. Neither Commistates nor the United States Atomic Energy Commission, nor any of their employees, nor any of their contractors, subcontractors, or their employees, makes uny warranty, express or implied; or assumes any

legal liability or responsibility for the accuracy any

pleteness or usefulness of any information, apparatus

product or process disclosed, would not infringe privately owned rights. 
CALCULATION OF CORRELATION EFFECTS FOR GRAIN-BOUNDARY AND DISLOCATION-PIPE SELF-DIFFUSION *

by

J. T. Robinson ${ }^{\star *}$ and N. L. Péterson ${ }^{\star \star *}$

\author{
Cornell University \\ Materials Science and Engineering \\ Ithaca, New York 14850 \\ $\star \star \star$ \\ Materials Science Division \\ Argonne National Laboratory \\ Argonne, Illinois 60439
}

\begin{abstract}
The correlation factor for grain-boundary and dislocation-pipe self-diffusion was calculated for a vacancy mechanism by assuming the applicability of the geometric model previously formulated by the authors. The correlation factor is temperature dependent and substantially lower than that for lattice selfdiffusion due to correlated vacancy motion. The calculations and experimental isotope effects for silver are consistent with a vacancy-dislocation (or grain boundary) binding energy that decreases 0.05-0.10 eV per atomic spacing away from the dislocation or grain boundary and many-body saddle-point interactions which are greater than those for lattice self-diffusion via vacancies.
\end{abstract}

This work was supported by the U. S. Atomic Energy Commisson. 


\section{INTRODUCTION}

The problem of resolving the mechanism for grain-boundary self-diffusion has received considerable attention during the past few years. The experiments of Cannon and Stark (1) and Volin, Lie, and Balluffi(2) and subsequent analysis by Balluffi(3) have shown that measurements of grain-boundary self-diffusion coefficients in face-centered-cubic ( $f c c$ ) metals are consistent with a vacancy mechanism. However, as in the case of lattice diffusion, measurements of only the self-diffusion coefficient do not conclusively determine the diffusion mechanism. Analysis of measurements of the isotope effect, $f \Delta K$, with respect to theoretical calculations of correlation factors are desirable for a more complete resolution of the problem. The isotope-effect parameters are defined as $f$ the correlation factor, and $\Delta \mathrm{K}$ the fraction of kinetic energy possessed by the diffusing atom in the saddle-point configuration in the jump direction.

Robinson and Peterson (4) have measured the isotope effect for grain-boundary self-diffusion in silver. Comparison of the $f^{\prime} \Delta K^{\prime}$ measurements with first-order calculations of the correlation factor was consistent with a vacancy mechamism for grain-boundary self-diffusion. [Note: The prime mark implies the parameters appropriate for grain-boundary diffusion.]

The major question regarding this analysis is the validity of the theoretical values of the correlation factor. The method of Leclaire and Lidiard (5) was employed with questionable validity, because the atomic geometry assumed near a grain boundary or dislocation does not yield atomic jumps along directions of two- 
fold rotational symmetry. Numerical results using the technique described gave correlation factors that were dependent upon the initial tracer jump. However, it was concluded that the isotopeeffect measurements and correlation-factor calculations were consistent with a vacancy mechanism for grain-boundary self-diffusion in fcc metals.

The purpose of the present paper is to report a more realistic calculation of the correlation factor for grain-boundary self-diffusion along dislocation cores that may be found in lowangle $\langle 100\rangle$ symmetric tilt boundaries. The geometric model is that proposed by Robinson and Peterson; ${ }^{(4)}$ the calculations are performed using Howard's ${ }^{(6)}$ general matrix method. The correlation factors that result from these calculations for a vacancy mechanism are compared with the experimental isotope effects for grainboundary self-diffusion in silver polycrystals and $\langle 100\rangle$ tilt boundaries. Since the results and subsequent interpretation are model dependent, the major properties of the geometric model are given.

\section{MODEL}

The atomic structure assumed for the compressed region of a dislocation in a $<100>$ low-angle symmetric tilt boundary is shown in Fig. 1. Details of the motivation for using such a structure are given in Ref. 4. The crosshatched circles represent sites along the last row of atoms in the extra, half plane of the edge dislocation. These are designated as type-1 sites and are expected to be positions of maximum vacancy-dislocation binding energy. (7) The other sites shown in Fig. 1 are type-2 sites and are nearest neigh- 
bors to the positions where the maximum binding energy occurs. The only other sites considered in the calculations are designated as type-3, which are nearest neighbors to type-2 sites. The vacancy-dislocation binding energy decreases with distance from type1 sites.

The vacancy-dislocation binding energy and the structure of the model imply that the atom-vacancy exchange frequencies are position dependent. Throughout the calculations it is assumed that atom-vacancy exchanges are possible only between nearest-neighbor positions. Therefore all tracer jumps. with nonzero $x$-displacement have identical $x$-projections. A jump dependent $x$-projection would change the value of the correlation factor; however, uncertainties in the atomic structure near a grain-boundary or dislocation disallow reasonable estimates of the position dependence of the tracer jump vectors. Tables 1 and 2 give the definitions of the vacancy jump frequencies and possible jump frequencies from the individual sites.

\section{METHOD OF CALCULATION}

Howard's method of calculating correlation factors is applicable to atomic jumps that are along directions of low crystal symmetry. Since the number of sites in the unit cell shown in Fig. 1 is small, all sites are considered unique. Furthermore, all tracer jumps with nonzero $x$-displacements are assumed to be unique. It should be noted that these assumptions of uniqueness are not absolutely necessary since some sites and jumps are equivalent. The equivalence relationships will only reduce the size of the matrices employed. 
The fundamental equation of the method is

$$
\mathrm{f}_{\mathrm{x}}=1+2 \hat{\mathrm{C}} \cdot \hat{\mathrm{T}}_{1}\left(\hat{\mathrm{I}}-\hat{\mathrm{T}}_{1}\right)^{-1} \cdot \hat{\mathrm{I}}
$$

where $f_{x}$ is the correlation factor due to atomic jumps with nonzero displacements in the $x$-direction. The term $\hat{c}$ is a row matrix whose $N$ components, $c_{\alpha}$, give the fraction of $\alpha$-type jumps in any long sequence of jumps, and $\mathrm{N}$ is the number of unique tracer jumps. The term $\hat{\mathrm{T}}_{1}$ is a $\mathrm{N}$ by $\mathrm{N}$ matrix with components

$$
t_{1}^{\alpha \beta}=p_{1+}^{\alpha \beta}-p_{1-}^{\alpha \beta}
$$

where $\mathrm{p}_{1 \pm}^{\alpha \beta}$ are equal to the probability that, given an initial $\alpha-$ type jump, the next tracer jump is a $\beta$ type and has an $x$-projection.. in the same (+) or opposite (-) direction as that of the initial jump. The term $\hat{I}$ is the $N$ by $N$ unit matrix, and $\hat{I}$ is the unit column matrix. The components $t_{1}^{\alpha \beta}$ are obtained by direci calculation of the $\mathrm{p}_{1 \pm}^{\alpha \beta}$

$$
\begin{array}{r}
\text { The terms } p_{1 \pm}^{\alpha \beta} \text { are given by } \\
\qquad p_{1 \pm}^{\alpha \beta}=p_{j \pm}^{\alpha} b_{j \pm}^{\beta},
\end{array}
$$

where $\mathrm{P}_{j \pm}^{\alpha}$ is the probability that, given an initial $\alpha$-type jump, the vacancy exists at a type-j site, which causes a $B$-type tracer jump with $x$-projection in the same $(+)$ or opposite $(-)$ direction as the $\alpha$ jump. The term $b_{j \pm}^{\beta}$ is the probability that the tracer and the vacancy at site $j$ exchange to give a $\beta$-type jump with the same $(+)$ or opposite (-) $x$-projection as the initial tracer jump. The $b_{j \pm}^{\beta}$ are obtained by inspection. The terms $F_{j \pm}^{\alpha}$ are $j^{\text {th }}$ components of $N$ column matrices, $\hat{\mathrm{P}}^{(\alpha)}$. The $\hat{\mathrm{p}}^{(\alpha)}$ are calculated from

$$
\hat{\mathrm{p}}^{(\alpha)}=(\hat{I}-\hat{A})^{-1} \cdot \hat{\mathrm{P}}_{\mathrm{O}}^{(\alpha)},
$$

where $\hat{A}$ is an $M$ dimensional square matrix with components $A_{i j}$ that 
give the probability that the next vacancy jump from site $j$ is to a type i site. The term $\hat{p}_{o}^{(\alpha)}$ is a M component column vector with components that give the vacancy distribution immediately after an a-type jump; which leaves the tracer at the origin.

By using Tables 1 and 2 in conjunction with. Fig. 1 , it is possible to determine the components of $\hat{A}$ and $\hat{c}$ in terms of $f i v e$ jump frequency ratios:

$$
\begin{aligned}
& \delta_{1}=\mathrm{w}_{3} / \mathrm{w}_{2} \\
& \delta_{2}=\mathrm{k} / \mathrm{w}_{2} \\
& \delta_{3}=\mathrm{w}_{2}^{\prime} / \mathrm{w}_{1} \\
& \delta_{4}=\mathrm{k}^{\prime} / \mathrm{w}_{1} . \\
& \delta_{5}=\mathrm{w}_{1} / \mathrm{w}_{2} .
\end{aligned}
$$

The only jumps that have nonzero $x$-displacements are of the type which place the tracer at $(0,0,0)$ and the vacancy at one of the sites given by $(1,0,1),(1,0,-1),(1,-1,0),(1,1,0),(-1,0,1)$, $(-1,0,-1),(-1,-1,0)$ and $(-1,1,0)$. The term $\hat{c}$ is given by. $c_{\Sigma} \hat{c}=\left[1 /\left(6+2 \delta_{5}\right), 1 /\left(2+6 / \delta_{5}\right), 1 /\left(6+2 \delta_{5}\right), 1 /\left(6+2 \delta_{5}\right), 1 /\left(6+2 \delta_{5}\right)\right.$, $\left.1 /\left(2+6 / \delta_{5}\right), 1 /\left(6+2 \delta_{5}\right), 1 /\left(6+2 \delta_{5}\right)\right]$,

where the factor $c_{\Sigma}$ accounts for the requirement that

$$
\sum_{\alpha=1}^{N} c_{\alpha}=1 \text {. }
$$

The matrix $\hat{A}$ is given by 


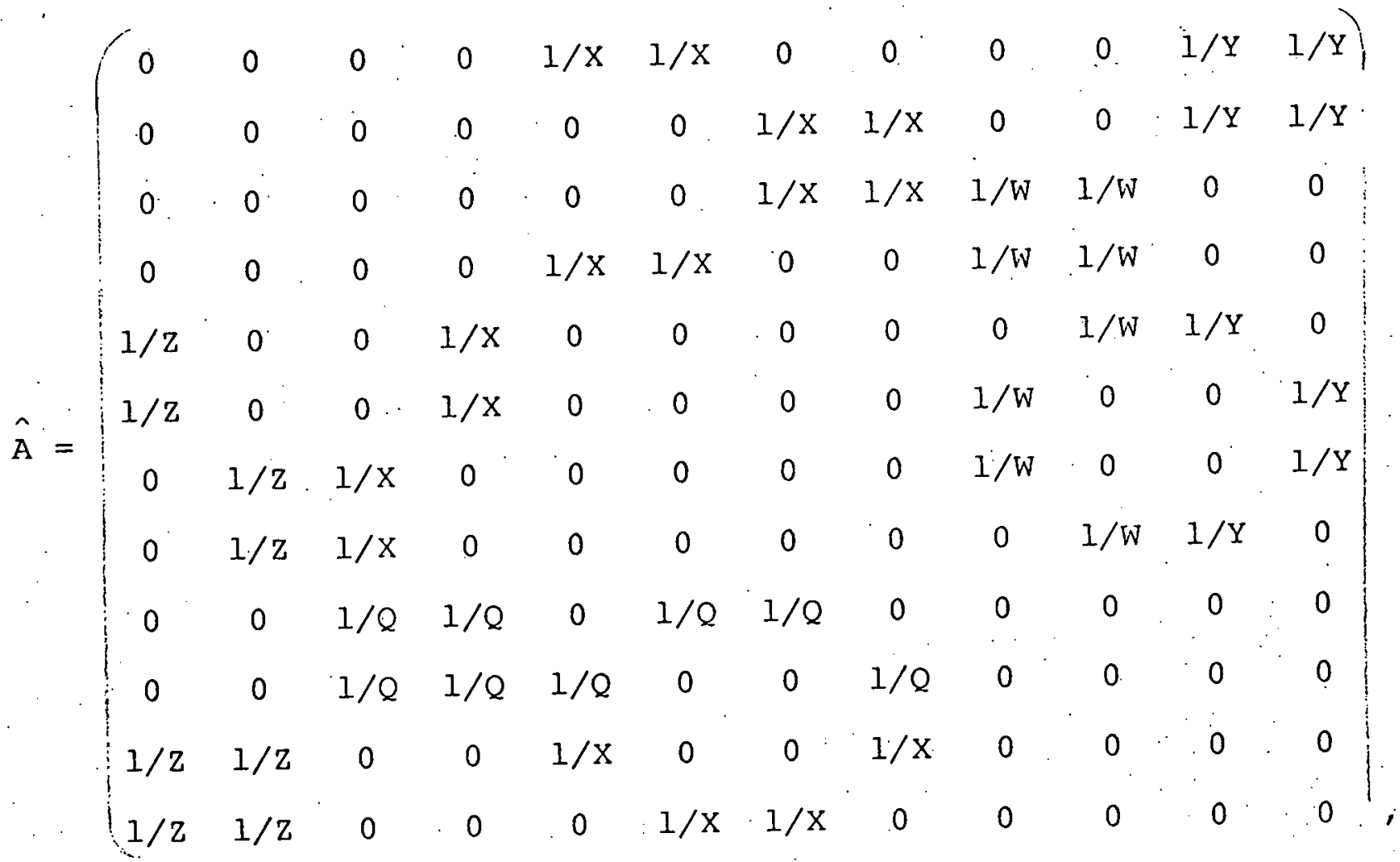

where $x=5+3 / \delta_{1}+4 \delta_{2} / \delta_{1}$

$$
\begin{aligned}
& Y=6+2 / \delta_{1}+4 \delta_{2} / \delta_{1} \\
& \mathrm{Z}=4+1 / \delta_{1}+7 \delta_{2} / \delta_{1} \\
& W=6+2 / \delta_{3}+4 \delta_{4} / \delta_{3} \\
& Q=3+5 \delta_{1}+4 \delta_{2} .
\end{aligned}
$$

The initial vacancy distribution aftex an $\alpha$-type jump corresponds to a vacancy at those equivalent sites possibly occupied by a vacancy after an $\alpha$-type jump has occurred. All components of $P_{0}(\alpha)$, $\alpha=1,2, \ldots 8$, are zero except the component corresponaing to the site occupied by the vacancy after the a jump places the tracer at the origin. In all cases, the value of the nonzero component is unity. Because all sites and jumps are considered unique, the $b_{j \pm}^{\beta}$ are probabilities that the vacancy jumps to the origin from sites giving a $\beta$-type jump. These nonzero terms are 


$$
\begin{aligned}
& \mathrm{b}_{11 \pm}^{1}=1 /\left(2+6 \delta_{1}+4 \delta_{2}\right) \\
& \mathrm{b}_{10 \pm}^{2}=1 /\left(2+6 \delta_{3}+4 \delta_{4}\right) \\
& \mathrm{b}_{8 \pm}^{3}=1 /\left(3+5 \delta_{1}+4 \delta_{2}\right) \\
& \mathrm{b}_{5 \pm}^{4}=1 /\left(3+5 \delta_{1}+4 \delta_{2}\right) \\
& \mathrm{b}_{12 \pm}^{5}=1 /\left(2+6 \delta_{1}+4 \delta_{2}\right) \\
& \mathrm{b}_{9 \pm}^{6}=1 /\left(2+6 \delta_{3}+4 \delta_{4}\right) \\
& \mathrm{b}_{7 \pm}^{7}=1 /\left(3+5 \delta_{1}+4 \delta_{2}\right) \\
& \mathrm{b}_{6 \pm}^{8}=1 /\left(3+5 \delta_{1}+4 \delta_{2}\right)
\end{aligned}
$$

The site-to-site vacancy-dislocation binding energy differences allow numerical evaluation of the jump frequency ratios given by Eq. (5). Assuming a constant migration energy and entropy factor,

$$
\frac{w_{i}}{w_{j}}=e^{-\left(\Delta E_{i}-\Delta E_{j}\right) / k_{B} T}
$$

where $w_{i}$ is the jump frequency, $\Delta E_{i}$ is the change in vacancy energy due to the jump specified by $w_{i}$, and $k_{B}$ and $T$ have their usual meanings. The term "binding energy" used throughout this paper is defined in the same sense as given in Ref.. 4 .

\section{RESULTS}

The results of the calculations of $f_{x}^{\prime}$ are given in Table 3. The binding conditions are chosen to represent a spectrum of values one might expect as the degree of correlation of vacancy motion changes.

The value of $\mathrm{f}_{\mathrm{x}}^{\prime}$ for the case of no vacancy-dislocation in- 
te'ractions is in good agreement with the value found by Leclaire and Iidiard for self-diffusion in fcc lattices. (5) The value of 0.818 compared with the true value of $0.782^{(8)}$ indicates that the model is restrictive in the sense that the number of near-neighbor shelis included is somewhat small. However, the finite size of the model introduces an inherent error of only $5 \%$. Because of correlated vacancy motion near the dislocation, the number of sites. considered would not be expected to be as important as in the case of lattice self-diffusion. Also, the results indicate that the temperature dependence and magnitude of the correlation factor are dependent upon the magnitude and rate of decrease of binding energy with distance from the dislocation core.

The results given in Table 3 are not expected to apply to all types of grain boundaries in fcc metals since the boundary structure is sensitive to the orientations of the boundary plane and single crystalline regions on either side of the boundary.

\section{DISCUSSION}

For a meaningful interpretation of the model and the results given in Table 3 , it is first necessary to relate the properties of the lattice self-diffusion isotope effect to those properties one could reasonably expect for grain-boundary and dislocationpipe self-diffusion.

Isotope-effect measurements for lattice self-diffusion in fcc material have established that diffusion occurs by means of a monovacancy mechanism, with possible divacancy contributions near the melting point. $(9-11)$. Also, $\Delta \mathrm{K}$ is independent of temperature and $0.85<\Delta \mathrm{K}<1$. Calculations of $\Delta \mathrm{K}$ by $\Lambda$ char ${ }^{(12)}$ agree with a temp- 


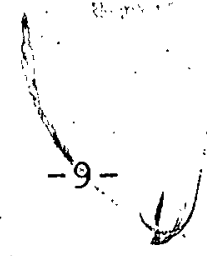

erature-independent $\Delta K$ and are. in reasonable agreement with the experimental results; $\Delta \mathrm{K}$ (calculated) for silver, is 0.80 and $\Delta \mathrm{K}$ (experimental) is 0.92 if divacancy contributions are negligible at low temperature cluded. (13)

We will assume that $\Delta \mathrm{K}^{\prime}$ for grain-boundary diffusion is similar to, but not identical with, $\Delta K$ for lattice diffusion. There is reason to believe that $\Delta K^{\prime}$ is somewhat smaller than $\Delta K$, and, if $\Delta K^{\prime}$ is temperature dependent, it decreases as the temperature increases. Near a grain boundary, lattice nearest-neighbor relations are not preserved, and the structure of the grain boundary is not close packed as in the lattice. The motions of saddle-point atoms in the lattice-diffusion process are constrained by nearest-neighbor interactions; therefore, $\Delta \mathrm{K}^{\prime}$ is near unity and temperature independent. It is possible that the vibrational amplitude of the atoms increases more with temperature in the open structure of grain boundaries than in the lattice. This would result in larger saddlepoint atom interactions with increasing temperature. Thus, a decrease in $\Delta \mathrm{K}^{\prime}$ as the temperature increases is not inconceivable. Àlso, an increase in saddle-point interactions in the diffusion direction would result in $\Delta \mathrm{K}^{\prime}<\Delta \mathrm{K}$ regardless of the temperature dependence. For the purpose of comparing the calculations of f' with expeximental values of $\mathrm{f}^{\prime} \Delta \mathrm{K}^{\prime}$, we will assume $\Delta \mathrm{K}^{\prime}$ is $\sim 0.75$. In Ref. 4, $\Delta K^{\prime}$ was assumed $\sim 0.85$; there is considerable uncertainty in this value of $\Delta K^{\prime}$ since the calculations of $f^{\prime}$ were questionable. The more realistic calculations given in this paper justify a lower $\Delta K^{\prime}$ if calculations of $f^{\prime}$ ' and estimates of $\Delta K^{\prime}$ reasonably approximate 
the experimental isotope effects.

Figure 2 shows the relationship between experimental isotope effects for silver grain-boundary self-diffusion ${ }^{(4)}$ and predicted isotope effects using the correlation factors from Table 3 and $\Delta K^{\prime}=0.75$. The dashed line represents the average experimental value of 0.46 for silver. [Note: Previously it was determined that to within experimental uncertainty the isotope effects were identical for grain-boundary self-diffusion in silver polycrystals and $16^{\circ}<100>$ symmetric tilt bicrystals (see Ref. 4). The same reasoning is implied throughout this paper.] Regardless of the estimated value of $\Delta K^{\prime}$, the binding condition $0.4,0.0$, and $0.0 \mathrm{eV}$ is unacceptable. From Table 3 and Fig. 2, this condition requires the nonphysical condition $\Delta K^{\prime}>1$. This confirms the deduction given in Ref. 4 that dislocation-pipe diffusion does not occur predominately by atomic motion along a single row of atoms in the dislocation core. The binding conditions $0.4,0.2$, and $0.1 \mathrm{eV}$ and $0.20,0.05$, and $0.05 \mathrm{eV}$ give unacceptable isotope-effect predictions. A $\Delta \mathrm{K}^{\mathrm{r}}>1$ is required at the lower temperature to match the calculated values to the experimental average.

The binding conditions $0.20,0.10$, and $0.0 .5 \mathrm{eV}$ and $0.20,0.15$, and $0.10 \mathrm{eV}$ both give physically acceptable values for $\Delta \mathrm{K}^{\prime}$. A temperature-dependent $\Delta K^{\prime}$, approximately linear in $(1 / T)$, gives $\Delta K^{\prime}=$ 0.70 at $623^{\circ} \mathrm{K}$, and $\Delta \mathrm{K}^{\prime}=0.76$ at $823^{\circ} \mathrm{K}$ if the correlation factor from the binding condition $0.20,0.15$, and $0.10 \mathrm{eV}$ is used to $\mathrm{fit}$ the average experimental isotope effect. A similar analysis for the binding condition $0.20,0.10$, and $0.05 \mathrm{eV}$ gives $\Delta \mathrm{K}^{\prime}=0.97$ at $623^{\circ} \mathrm{K}$, and $\Delta \mathrm{K}^{\prime}=0.85$ at $823^{\circ} \mathrm{K}$. Thus, it is apparent that a unique bindjing 
condition and temperature-dependent f' cannot be determined without further knowledge of the magnitude of $\Delta \mathrm{K}^{\prime}$.

However, the nonuniqueness of calculated $f^{\prime}$ does not substantially distract from the essential features of grain-boundary and dislocation-pipe diffusion. The binding conditions 0.20, 0.15, and $0.10 \mathrm{eV}$ and $0.20,0.10$, and $0.05 \mathrm{eV}$ are not substantially different. Both give the correlation factors significantly lower than those for lattice self-diffusion in fcc lattices. Within the framework of the geometric model, the exact calculations of $f^{\prime}$ prove that the vacancy-dislocation binding energy decreases $0.05-0.10 \mathrm{eV}$ per nearest-neighbor spacing away from the dislocation or grain boundary in the grain boundaries in silver where the isotope-effect measurements apply. It should be noted that the values of $f$ ' which result fxom the binding condition $0.20,0.15$, and $0.10 \mathrm{ev}$ are preferred because a more reasonable $\Delta K^{\prime}$ ' results.

Inasmuch as the geometric model restricts tracer and vacancy motion to a small region near the dislocation or grain boundary, the calculation of $f^{\prime}$ gives the effective grain-boundary width or dislocation core diameter for diffusion. The agreement shown in Fig. 2 for calculated $f^{\prime}$, predicted $\Delta K^{\prime}$, and experimental isotope effects of grain boundaries in silver implies that fast self-diffusion occurs within two to four lattice parameters around a grain boundary or dislocation. 


\section{REFERENCES}

1. R. F. Cannon and J! P., Stark, J. Appl. Phys. 40, 4361 (1969).

2. T. E. Volin, K. H. Lie, and R. W. Balluffi, Acta Met. 19, $263(1971)$.

3. R. W. Balluffi, Phys. Status Solidi 42, 11 (1970).

4. J. T. Robinson and N. L. Peterson, Surface Science 31, 586 (1972).

5. A. D. LeClaire and A. B. Lidiard, Phil. Mag. 1, 518 (1956).

6. R. E. Howard, Phys. Rev. 144,650 (1966).

7. M. Doyama and R.M.J. Cotterill, Suppl. Japan Inst. Metals 9 , $55(1968)$.

8. K. Compaan and Y. Haven, Trans. Faraday Soc. 52, 786 (1956).

9. N. L. Peterson, Phys. Rev. 136, A568 (1964).

10. S.J. Rothman and N. L. Peterson, Phys. Status Solidi 35, $305(1969)$.

11. S. J. Rothman, N. L. Peterson, and J. T.Robinson, Phys. Status Solidi 39,635 (1970).

12. B. N. Narahari Achar, Phys. Rev. B2, 3848 (1970).

13. H. Mehrer and A. Seeger, Phys. Status Solidi 39;646 (1970). 
Table 1. Definitions of vacancy jump frequencies

\begin{tabular}{|ccc|}
\hline $\begin{array}{c}\text { Initial site } \\
\text { type }\end{array}$ & $\begin{array}{c}\text { Final site } \\
\text { type }\end{array}$ & $\begin{array}{c}\text { Jump frequericy } \\
\text { designation }\end{array}$ \\
\hline 1 & 1 & $w^{\prime} 1$ \\
1 & 2 & $w^{\prime}$ \\
2 & 1 & $w_{2}$ \\
1 & $3^{a}$ & $\mathrm{k}^{\prime}$ \\
2 & 3 & $\mathrm{k}$ \\
2 & 2 & $\mathrm{w}_{3}$ \\
\hline
\end{tabular}

a The site 3 here refers to positions in the dilatation region of the dislocation that can be reached from a type-l site. The binding of a vacancy at these sites is assumed to be identical to that for type-3 sites in the compression region. 
Table 2. Vacancy jump frequencies.

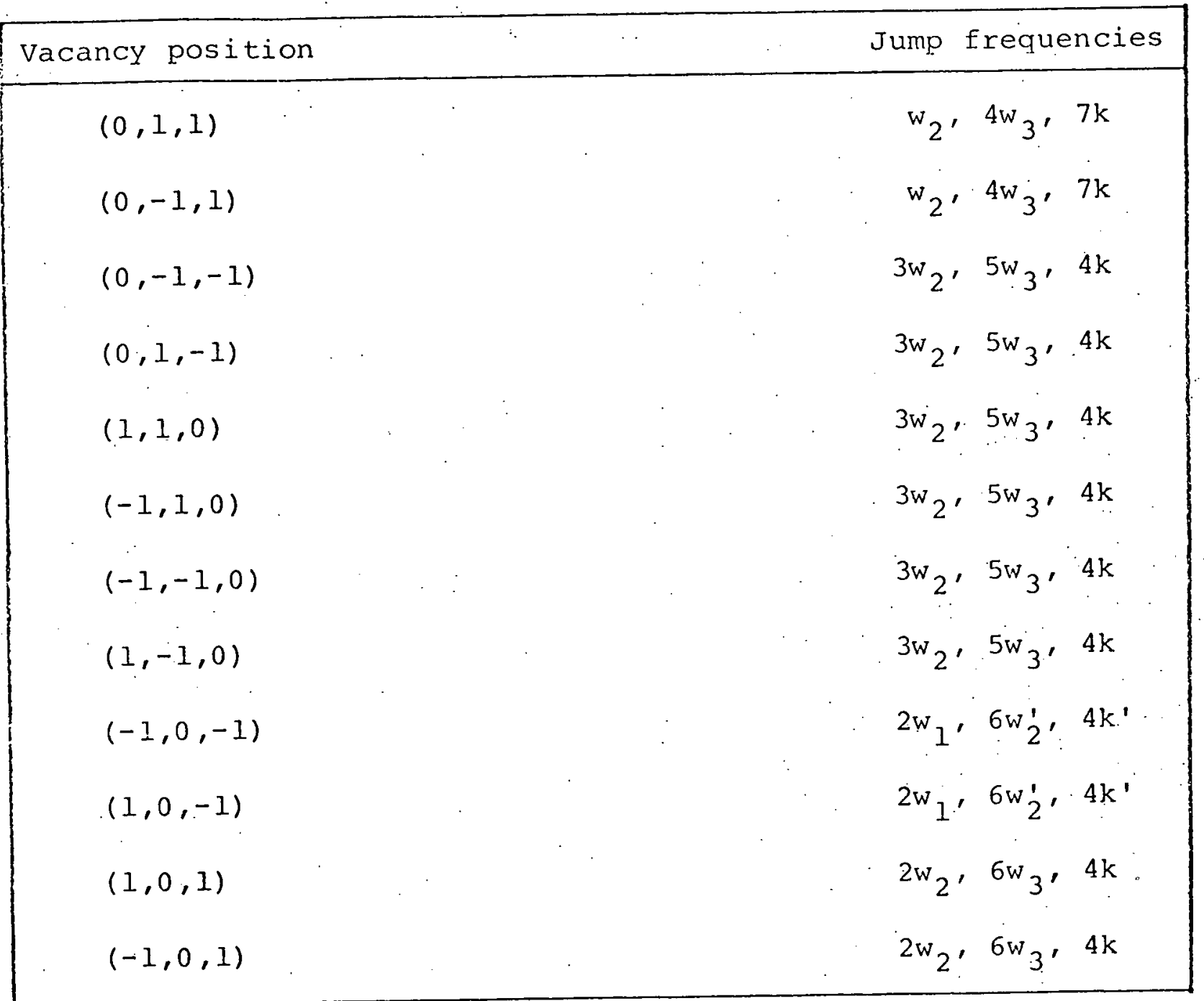


Table 3. Temperature and binding energy dependence of calculated correlation factors

\begin{tabular}{|c|ccccc|}
\hline $\begin{array}{c}\text { Binding } \\
\text { conditions } \\
(\mathrm{eV})\end{array}$ & \multicolumn{4}{|c}{$\mathrm{f}^{\prime}$ at the indicated temperature, ${ }^{\circ} \mathrm{K}$} \\
\cline { 2 - 6 } $0.00,0.00,0.00$ & 623 & 673 & 723 & 773 & 823 \\
$0.20,0.15,0.10$ & 0.818 & 0.818 & 0.818 & 0.818 & 0.818 \\
$0.20,0.10,0.05$ & 0.609 & 0.624 & 0.638 & 0.649 & 0.660 \\
$0.20,0.05,0.05$ & 0.472 & 0.491 & 0.510 & 0.527 & 0.543 \\
$0.40,0.20,0.10$ & 0.420 & 0.438 & 0.455 & 0.472 & 0.488 \\
$0.40,0.00,0.00$ & 0.435 & 0.445 & 0.464 & 0.478 & 0.491 \\
\hline
\end{tabular}




\section{FIGURE CAPTIONS}

Figure 1. Structural model for correlation-factor calculations.

Figure 2. Temperature dependence of the isotope effect, calculated using values from Table 3 and $\Delta \mathrm{K}^{\prime}=0.75$, - and o are experimental data from Ref. 4 for polycrystals and $\langle 100\rangle$ tilt bicrystals of silver, --average of the experimental data from Ref. 4. 


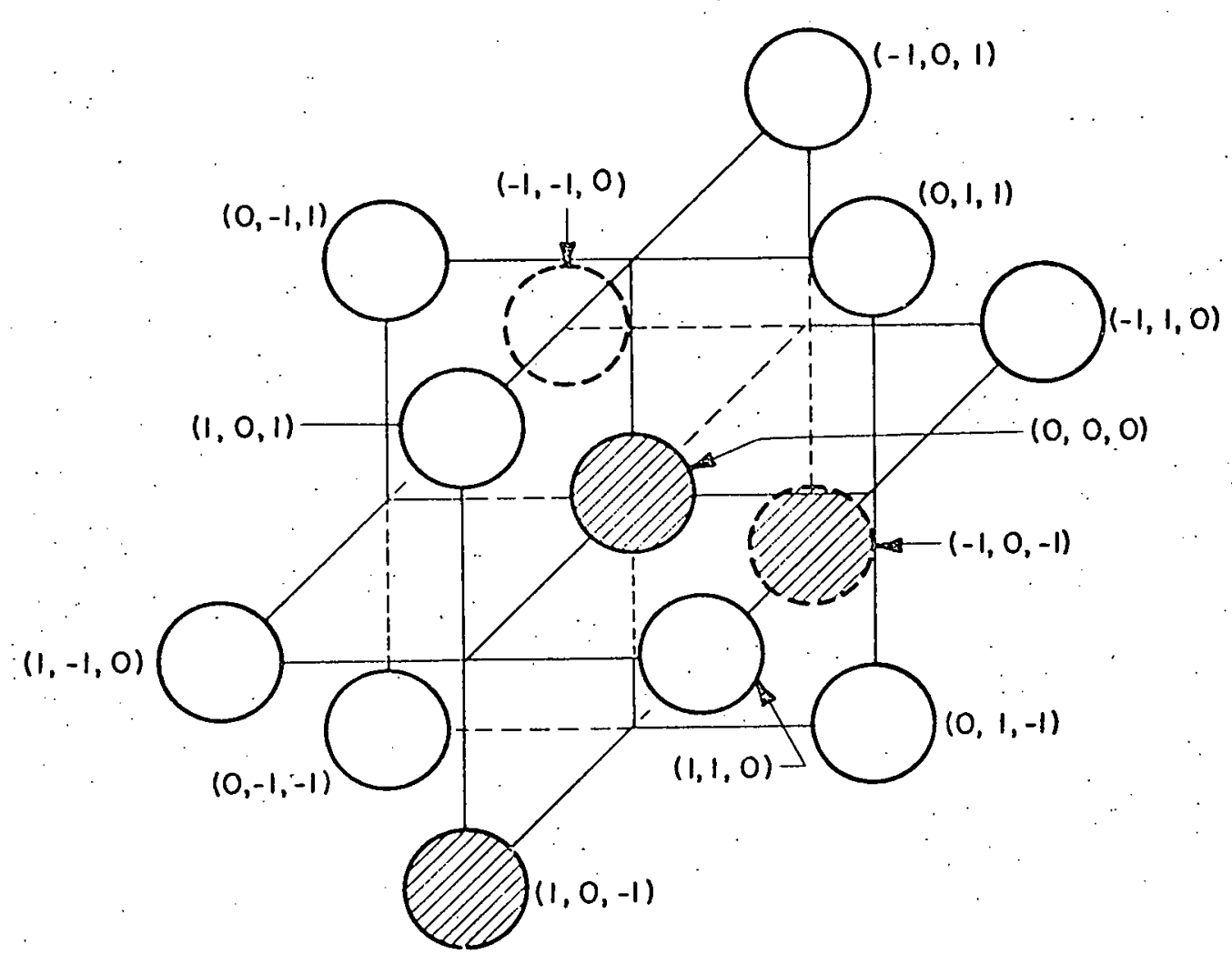

Figure 1 


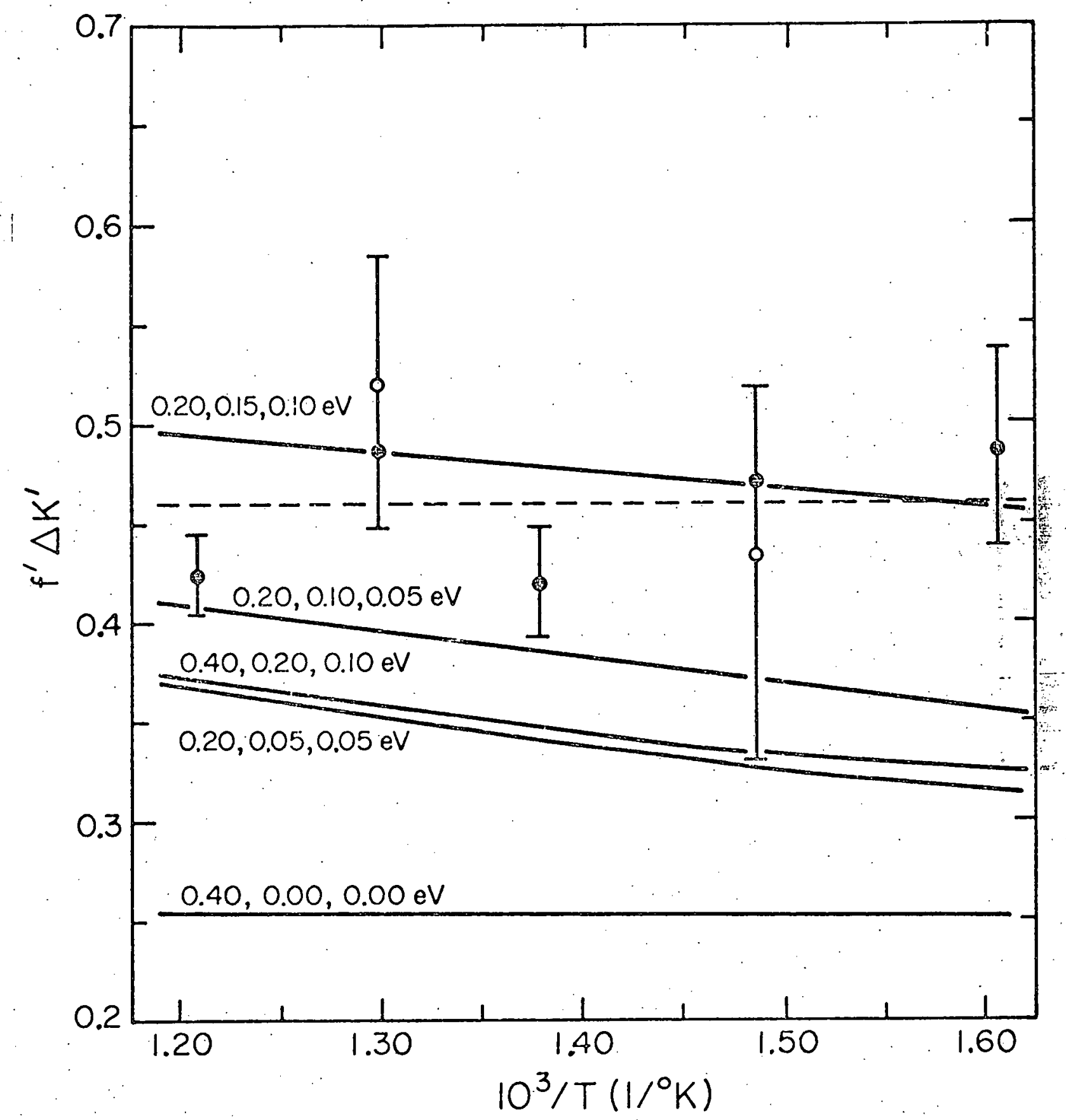

Figure 2 